\title{
Is Dietary Milk Intake Associated with Cataract Extraction History in Older Adults? An Analysis from the US Population
}

\author{
Osama M. Mustafa and Yassine J. Daoud (i) \\ Cornea, Cataract, and Refractive Surgery Division, The Johns Hopkins University Wilmer Eye Institute, Baltimore, \\ MD 21287, USA \\ Correspondence should be addressed to Yassine J. Daoud; ydaoud1@jhmi.edu
}

Received 27 September 2019; Revised 15 January 2020; Accepted 18 January 2020; Published 20 February 2020

Academic Editor: Mohit Parekh

Copyright () 2020 Osama M. Mustafa and Yassine J. Daoud. This is an open access article distributed under the Creative Commons Attribution License, which permits unrestricted use, distribution, and reproduction in any medium, provided the original work is properly cited.

\begin{abstract}
Background. Galactose accumulation in the lens tissue is known to be cataractogenic. Whether consistent dietary intake of lactose-which consists of glucose and galactose-predisposes to senile cataract remains unclear. This study was conducted to investigate the association between a number of dietary milk intake indicators and cataract extraction history in a representative sample of older adults from the US population. Methods and Materials. This is a cross-sectional, population-based study. Participants of the United States National Health and Nutrition Examination Survey 2001-2008 who were $\geq 50$ years old and provided a complete history of their usual daily dietary intake were included. Exclusion criteria were special diets, extreme daily energy intake, and missing outcome (i.e., cataract extraction history). Indicators of milk intake used were early-life intake regularity, current daily milk/total dairy intake amounts, and estimated lifelong milk exposure. Odds ratios (OR) and 99\% confidence intervals (99\% CI) were calculated with fitting weights to better represent the population-based estimates. Results. Among the 5930 studied participants, early-life milk intake regularity was not associated with cataract extraction history in age/ sex/ethnicity-adjusted and multivariable-adjusted models ( $p$ trend $=0.064$ and 0.094 , respectively). Current daily milk intake was associated with a slight reduction in the likelihood of cataract extraction in the age/sex/ethnicity-adjusted model $(\mathrm{OR}=0.885 \mathrm{per}$ cup equivalents, $99 \% \mathrm{CI}=0.795-0.986)$ and in the multivariable model ( $\mathrm{OR}=0.871$ per cup equivalents, $99 \% \mathrm{CI}=0.746-0.993)$. However, no such association was observed between quartiles of current dietary milk intake and cataract extraction history ( $p=0.154$ and $p=0.317$ for age/sex/ethnicity-adjusted and multivariable-adjusted models, respectively). Neither current total dairy intake nor estimated lifelong milk exposure was significantly associated with the outcome in the final multivariable models. Conclusion. There appears to be no direct relationship between several indicators of dietary milk consumption and cataract extraction history in the general American population.
\end{abstract}

\section{Introduction}

Several mechanisms, such as oxidative and osmotic stress, mediate cataract development. In states of oxidative stress, the production of free radicals exceeds the tissue's elimination capacity, resulting in the deposition of alpha-crystallin and impairment of lens transparency. In osmotic stress states, excess sugars (e.g., galactose) are converted to their sugar alcohol counterparts (e.g., galactitol), the accumulation of which disturbs water and electrolyte homeostatic mechanisms necessary to maintain tissue transparency [1]. This osmotic imbalance is believed to underlie the early development of cataract in inborn disorders of galactose metabolism, such as galactokinase deficiency and galactosemia [2]. In addition to the direct osmotic damage caused by its accumulation, recent evidence suggests that galactose could accelerate lens epithelial cell senescence in vitro, possibly by impairing autophagy and mitochondrial function [3]. The accumulation of sugar alcohols is also believed to play a role in hyperglycemia- and diabetes-induced cataracts [4].

Because of the modulatory role of diet in disease outcomes, and the cataractogenic potential of galactose, researchers speculated that long-term dietary intake of 
galactose might be associated with senile cataract formation [5]. In human diet, the major source of galactose is lactose in milk and dairy products. To date, the effect of dietary milk consumption on cataract development remains controversial. Early reports from regions of Asia concluded that possible links exist $[5,6]$. However, recent investigations in European and Mediterranean populations were less conclusive $[7,8]$. In this study, we examined the relationship between indicators of dietary milk exposure and cataract extraction history in a nationally representative sample of the American population.

\section{Materials and Methods}

2.1. Study Design and Population. This is a cross-sectional analysis of nationally representative data of adults, aged 50 years and over, participating in the National Health and Nutrition Examination Survey (NHANES). The protocols were approved by the National Center for Health Statistics Research Ethics Review Board and conducted in line with the tenets of the Declaration of Helsinki. Participants who provided a complete first-day dietary survey from 2001 through $2008(n=37521)$ were eligible for inclusion in this analysis if they were $\geq 50$ years old and considered the detailed $24 \mathrm{~h}$ food intake as representative of their usual intake $(n=6904)$. Eligible participants on special diets $(n=927)$, with extreme daily total energy intake ( $\geq 3$ standard deviations above or below the log-transformed sample mean) [9] $(n=44)$, and/or additionally missing the outcome variable $(n=3)$ were excluded. Therefore, a total of 5930 participants were included in this analysis.

2.2. Milk Intake Assessment and Indicators. Early-life daily milk intake regularity was reported for three age intervals: $5-12,13-17$, and 18-35 years of age. Participants reporting milk drinking "once a day or more" during all three intervals were considered to be always-regular daily milk drinkers from childhood through early adulthood. Current daily milk intake (and total dairy intake) was assessed using 24-hour dietary recall using United States Department of Agriculture (USDA) automated multiple-pass method [10]. The servings (in cup equivalents) were determined using MyPyramid Equivalent Databases and Food Patterns Equivalent Databases relevant to each of the NHANES cycles [11]. The participant-specific amounts of current daily milk intake and total dairy intake were assessed on a continuous scale as well as in sample-based quartiles. Self-perceived consistency of milk intake was identified by asking the participants whether they had been drinking milk at least 5 times a week for most/ all of their life.

Since tracing lifelong exposure to a particular dietary component often proves difficult, the attempt was made to evaluate the lifelong/long-term milk exposure using a proxy indicator that categorizes participants based on combinations of consistency and amounts of early-life and current milk intake, respectively (Table S1, Supplementary Materials). The high exposure group was defined by participants who were always-regular early-life daily milk drinkers and had highest quartile of current milk intake, while the low exposure group consisted of the participants who were never regular early-life milk drinkers and were of the lowest current milk intake quartile group. The categorization of remaining groups is shown in Table S1.

2.3. Outcome and Confounding Factors. The outcome of interest in this study was cataract extraction history. To control for demographic and lifestyle variables that may confound the relationship between the examined indicators and the outcome, we adjusted for age, sex, and ethnicity in the baseline models. In addition, educational attainment, income [12], health insurance status, smoking status, alcohol drinking history, body mass index (BMI), diabetes history, and hypertension history were adjusted for in the multivariable models. A positive history of diabetes was identified by the self-report of physician-diagnosed disease and/or by taking diabetic medications/insulin at the time of survey. Hypertension was defined by self-report of diagnosis by a physician or other health professional at two different visits or by taking antihypertensive medications at the time of survey. BMI was calculated as the weight by height $\left(\mathrm{kg} / \mathrm{m}^{2}\right)$. Furthermore, total energy intake (kcal) was controlled for in analyses that included current dietary milk and total dairy intakes, including lifelong milk exposure.

2.4. Statistical Analysis. Characteristics of the study sample and milk consumption indicators were summarized as weighted means and percentages for continuous and categorical variables, respectively. The relationship between milk-based predictors and outcome was initially explored univariably as well as with adjustments of age, sex, and ethnicity. Then, potential confounding factors were collectively controlled for to produce the final multiple logistic regression models. The analysis was conducted using SPSS CS v.22 (IBM Corp, Armonk, NY). Odds ratios and 99\% confidence intervals ( $99 \% \mathrm{CI})$ were calculated and the corresponding alpha error rate of $<0.01$ (two-tailed) was considered for statistical significance. To better reflect the US national population estimates, the data were analyzed using appropriate weights to account for survey nonresponse, group oversampling, and dietary intake variation associated with the complex structure of the survey [13].

\section{Results}

The sociodemographic characteristics of the study population are shown in Table 1. The mean age of participants was $63.8 \pm 9.9$ years, of whom $15.7 \%$ had a positive history of cataract extraction. On average, participants reported current daily consumption of $0.91 \pm 1.14$ and $1.43 \pm 1.39$ cup equivalents/day of milk and total dairy products, respectively, at the time of the survey (Table 2). About $17 \%$ and $8 \%$ were estimated to have had high and low lifelong exposure to milk, respectively.

3.1. Cataract and Early-Life Milk Consumption. When using daily (i.e., $\geq 7$ times a week) milk drinking as an indication of 
TABLE 1: Sociodemographic and baseline characteristics of the study population.

\begin{tabular}{|c|c|}
\hline & $\begin{array}{l}\text { Summary } \\
\text { finding }\end{array}$ \\
\hline Age (year), mean \pm SD & $63.8 \pm 9.9$ \\
\hline $\begin{array}{l}\text { Sex }(\%) \\
\text { Male } \\
\text { Female } \\
\end{array}$ & $\begin{array}{l}49.0 \\
51.0\end{array}$ \\
\hline $\begin{array}{l}\text { Race/ethnicity (\%) } \\
\text { Mexican American, other Hispanic, other } \\
\text { race } \\
\text { Non-Hispanic white } \\
\text { Non-Hispanic black }\end{array}$ & $\begin{array}{c}11.5 \\
80.4 \\
8.2\end{array}$ \\
\hline $\begin{array}{l}\text { Level of education (\%) } \\
<9^{\text {th }} \text { grade } \\
9^{\text {th }}-11^{\text {th }} \text { grade } \\
\text { High school or equivalent } \\
\text { Some college education or AA degree } \\
\text { College graduate or higher } \\
\end{array}$ & $\begin{array}{c}9.7 \\
11.2 \\
28.0 \\
25.8 \\
25.2\end{array}$ \\
\hline $\begin{array}{l}\text { Income to poverty ratio }(\%) \\
\text { Below } 100 \% \\
100 \%-300 \% \\
\text { More than } 300 \% \\
\end{array}$ & $\begin{array}{c}8.9 \\
38.6 \\
52.5\end{array}$ \\
\hline $\begin{array}{l}\text { Current health insurance (\%) } \\
\text { Yes } \\
\text { No }\end{array}$ & $\begin{array}{c}91.5 \\
8.5 \\
\end{array}$ \\
\hline $\begin{array}{l}\text { Smoking status }(\%) \\
\text { Nonsmoker } \\
\text { Current or former smoker }\end{array}$ & $\begin{array}{l}47.3 \\
52.7 \\
\end{array}$ \\
\hline $\begin{array}{l}\text { Alcohol consumption history (\%) } \\
\text { Lifelong abstinent } \\
\text { No history of binge drinking } \\
\text { Positive history of binge drinking }\end{array}$ & $\begin{array}{l}14.2 \\
71.6 \\
14.2 \\
\end{array}$ \\
\hline $\begin{array}{l}\text { Diabetes mellitus history (\%) } \\
\text { Positive } \\
\text { Negative } \\
\end{array}$ & $\begin{array}{l}11.6 \\
88.4\end{array}$ \\
\hline $\begin{array}{l}\text { Hypertension history (\%) } \\
\text { Positive } \\
\text { Negative }\end{array}$ & $\begin{array}{l}43.8 \\
56.2\end{array}$ \\
\hline $\begin{array}{l}\text { Body mass index }\left(\mathrm{kg} / \mathrm{m}^{2}\right), \% \\
(\text { Mean } \pm \text { SD) } \\
\text { Underweight and normal weight }(<25) \\
\text { Overweight }(25 \text { to }<30) \\
\text { Obese }(\geq 30) \\
\end{array}$ & $\begin{array}{c}(28.3 \pm 5.9) \\
30.0 \\
38.8 \\
31.3\end{array}$ \\
\hline $\begin{array}{l}\text { Had cataract extraction }(\%) \\
\text { Yes } \\
\text { No }\end{array}$ & $\begin{array}{l}15.7 \\
84.3\end{array}$ \\
\hline
\end{tabular}

regular milk drinking in early life, $44.5 \%$ of participants were considered regular daily milk drinkers throughout the childhood to early adulthood period (i.e., 5-35 years) (Table 2). Over a third (35.1\%) of participants were less regularly drinking milk during one or more of the early-life intervals (i.e., 5-12, 13-17, and 18-35 years) and, thus, were considered to have a variable regular daily intake during the childhood to early adulthood period. The remaining $20.3 \%$, who previously reported never being regular milk drinkers most or all of their life, were considered to have never had regular milk intake during childhood to early adulthood period. Neither univariable nor multivariable examination showed a statistically significant association between early-life milk intake and cataract extraction history (Table 3 ).

3.2. Cataract and Current Daily Milk Intake. The median number of cup equivalents of daily milk intake was 0.53 (IQR $=0.12-1.27)$ at the time of the survey. Univariable analysis did not show a significant association between current daily intake and cataract extraction history $(\mathrm{OR}=0.970$ per cup equivalent, 99\% CI=0.891-1.055; $p=0.336)$. In contrast, age/sex/ethnicity-adjusted and multivariable models suggested a reduction in the risk of cataract extraction history with the reported current milk intake $(\mathrm{OR}=0.885$ per cup equivalent, $99 \%$ $\mathrm{CI}=0.795-0.986, \quad p=0.004$ and $\mathrm{OR}=0.871$ per cup equivalent, 99\% CI $=0.746-0.993, p=0.007$, respectively). However, when examining participants in quartiles of current daily milk intake, it was not found to be associated with cataract extraction history in the studied population (Table 3).

3.3. Cataract and Current Total Dairy Intake. The median number of cup equivalents of total dairy product intake was $1.07(\mathrm{IQR}=0.44-2.02)$ at the time of the survey. Univariable examination suggested a statistically significant decrease in the odds of cataract extraction history with increased intake $(\mathrm{OR}=0.891,99 \% \mathrm{CI}=0.825-0.963, p<0.001)$. However, this association was not found to be significant after adjusting for age and other confounding factors (age/gender/ethnicity-adjusted $\mathrm{OR}=0.914,99 \% \mathrm{CI}=0.815-1.026$, $p=0.042$ and multivariable-adjusted $\mathrm{OR}=0.910,99 \%$ $\mathrm{CI}=0.798-1.039, p=0.063$, respectively). Likewise, examination of the relationship between quartiles of total dairy intake and cataract extraction history did not show any significant findings (Table 3 ).

3.4. Cataract and Lifelong Milk Exposure. No significant univariable association was observed between lifelong milk exposure and cataract extraction history $(p=0.543)$. Moreover, although the model adjusting for age, gender, ethnicity, and total energy intake suggested a significant trend between lifelong milk exposure and cataract extraction history $(p=0.002)$, none of the individual groups were significantly different from the high exposure group ( $p>0.01$, Table 3$)$. Also, after adjusting for all potential confounders, neither the trend of association nor the individual groups met the criterion of significance in this study (Table 3).

3.5. Cataract and Self-Perceived Consistency of Milk Consumption. Approximately $45.4 \%$ perceived themselves as consistent milk drinkers ( $\geq 5$ times/week) for most or all of their life, whereas $20.2 \%$ said that they never consumed milk consistently (Table 2). Univariate examination revealed no significant association between self-perceived intake consistency and cataract extraction history $(\mathrm{OR}=1.088,99 \%$ $\mathrm{CI}=0.827-1.432$ and $\mathrm{OR}=0.956,99 \% \mathrm{CI}=0.751-1.216$ for the never-consistent and sometimes-consistent groups, 
TABLE 2: Summary statistics of milk consumption indicators, including usual daily intake amounts (in cup equivalents) of milk and total dairy products, and the estimated level of lifelong milk exposure by participants of $24 \mathrm{~h}$ dietary survey.

\begin{tabular}{|c|c|c|c|c|}
\hline & Total & $\begin{array}{l}\text { Positive cataract extraction } \\
\text { history (\% within-group) }\end{array}$ & $\begin{array}{l}\text { Negative cataract extraction } \\
\text { history (\% within-group) }\end{array}$ & $\begin{array}{c}\text { Univariate } p \\
\text { value }\end{array}$ \\
\hline $\begin{array}{l}\text { Current total milk intake (cup } \\
\text { equivalents/day), mean } \pm S D\end{array}$ & $0.91 \pm 1.14$ & $0.88 \pm 0.95$ & $0.92 \pm 1.17$ & 0.336 \\
\hline $\begin{array}{l}\text { Current total dairy intake (cup } \\
\text { equivalents/day), mean } \pm \text { SD }\end{array}$ & $1.43 \pm 1.40$ & $1.26 \pm 1.13$ & $1.46 \pm 1.44$ & $<0.001$ \\
\hline \multicolumn{5}{|c|}{ Early-life regular daily milk drinking (\%) } \\
\hline $\begin{array}{l}\text { Self-reported as never a regular } \\
\text { milk drinker }\end{array}$ & 20.3 & 22.0 & 20.10 & \\
\hline Variable (sometimes daily) & 35.1 & 32.8 & 35.60 & 0.224 \\
\hline Always-regular daily milk drinker & 44.5 & 45.2 & 44.40 & \\
\hline \multicolumn{5}{|l|}{ Estimated lifelong milk exposure (\%) } \\
\hline Low & 7.9 & 6.5 & 8.1 & 0.543 \\
\hline Below average & 20.3 & 21.2 & 20.2 & \\
\hline Average & 29.3 & 29.2 & 29.3 & \\
\hline Above average & 25.4 & 25.3 & 25.4 & \\
\hline High & 17.1 & 17.8 & 17.0 & \\
\hline \multicolumn{5}{|c|}{ Self-perceived consistency ( $\geq 5 /$ week) of milk drinking throughout life (\%) } \\
\hline Yes & 45.4 & 45.3 & 45.4 & 0.476 \\
\hline Sometimes but not always & 34.4 & 33.0 & 34.6 & \\
\hline Never & 20.2 & 21.7 & 20.0 & \\
\hline
\end{tabular}

respectively, compared to the consistent group; $p$ trend $=0.476)$. After adjustment for age, gender, and ethnicity, the association was statistically significant $(p$ trend $=0.004)$, with higher odds in participants who reported variable/sometimes-consistent milk drinkers compared to consistent milk drinkers (Table 3). However, this relationship was not found to be significant after controlling for potential confounding variables (Table 3).

\section{Discussion}

The potential role of galactose in cataract development was first identified in 1935 in rats that were fed a high-galactose diet [14]. A decade later, the observation of cataract development in children with inborn galactose metabolism errors was made [15]. Because of the known connection between galactose metabolism and cataractogenesis, researchers postulated that the long-term, high intake of milk products might cause subliminal but cumulative damage to the lens, leading to senile cataract formation [16]. Some even suggested decreasing dietary intake of milk and dairy products or using inhibitors of pertinent enzymes to treat predisposed individuals $[17,18]$. In this study, we explored the association between several indicators of dietary milk consumption and cataract extraction history in older adults. To our knowledge, the relationship between life-long milk consumption and cataract has not been explored previously in a representative sample of the general US population.

While the link between galactose-mediated damage and congenital cataract in galactosemic patients is well-established, the evidence of a relationship between milk consumption and age-related cataract in normal individuals has been, at best, inconclusive. Earlier epidemiological studies suggested that a relationship might exist $[5,6,19]$. In one study, the geographic distribution of milk consumption and lactose malabsorption in relation to the incidence of cataract was used to imply a possible link [5]. In another, self-reported daily milk intake was compared between cases of cataract and consecutive healthy controls in ophthalmic clinic patients [6]. The authors found that higher milk intake was positively correlated with cortical cataracts [6].

However, more recent studies have found no such association between milk intake and cataract risk. For example, case-control studies did not find a significant difference in cataract [7] or cataract extraction risk [20] based on milk and dairy product consumption. Likewise, a recently published prospective study of adult Mediterranean subjects with high cardiovascular risk found no association between cataract incidence and total dairy products intake, including milk [8]. These findings are consistent with what we observed in this large, population-based study. We attempted to examine the possibility that individuals with consistently higher lactose levels accrue greater damage over long years of milk consumption [21] and found it to be insignificant. We also explored the possibility of such an effect being specifically related to consumption in the early years of a participant's life (i.e., 5-35 years) and similarly found no significant association.

A possible explanation for the lack of direct association is that hypergalactosemia alone may be insufficient to cause damage to the lens in healthy individuals. As some empirical evidence suggests, immediate susceptibility combined with high-galactose intake is needed to affect the lens. This notion is consistent with the synergistic effect observed when two cataractogenic factors are introduced simultaneously in an animal model [22]. For example, an investigation of the effect of high milk intake in Sprague-Dawley rats showed that the presence of underlying damage to the lens (e.g., by naphthalene) led to significant susceptibility to injury by excessive milk intake [23]. This notion is also in line with 
TABLE 3: The odds ratio estimates of several indicators of milk intake in relation to cataract extraction history in a sample of $\geq 50$-year-old participants of the National Health and Nutrition Examination Survey 2000-2008.

\begin{tabular}{|c|c|c|c|c|}
\hline & \multicolumn{2}{|c|}{$\begin{array}{l}\text { Age, gender, and ethnicity- } \\
\text { adjusted* }\end{array}$} & \multicolumn{2}{|c|}{ Multivariable } \\
\hline & OR $(99 \% \mathrm{CI})$ & $p$ value & OR $(99 \% \mathrm{CI})$ & $p$ value \\
\hline Early-life daily milk consumption & & $p=0.064$ & & $p=0.094$ \\
\hline Never regular daily milk drinker & $1.250(0.925-1.690)$ & 0.053 & $1.332(0.943-1.881)$ & 0.031 \\
\hline Variable (sometimes daily) & $1.240(0.938-1.638)$ & 0.045 & $1.158(0.825-1.624)$ & 0.255 \\
\hline Regular daily milk drinker & Reference & - & Reference & - \\
\hline Current milk intake & $p=0.154$ & & $p=0.317$ & \\
\hline Q1 & $1.262^{\top}(0.918-1.734)$ & 0.057 & $1.272(0.893-1.812)$ & 0.076 \\
\hline Q2 & $1.299(0.856-1.971)$ & 0.10 & $1.267(0.799-2.009)$ & 0.178 \\
\hline Q3 & $1.037(0.764-1.406)$ & 0.754 & $1.120(0.797-1.575)$ & 0.379 \\
\hline Q4 & Reference & - & Reference & - \\
\hline Current total dairy intake & $p=0.836$ & & $p=0.528$ & \\
\hline Q1 & $1.268(0.891-1.804)$ & 0.078 & $1.251(0.910-1.72)$ & 0.165 \\
\hline Q2 & $1.123(0.769-1.642)$ & 0.418 & $1.202(0.863-1.674)$ & 0.272 \\
\hline Q3 & $1.097(0.801-1.503)$ & 0.437 & $1.138(0.890-1.454)$ & 0.296 \\
\hline Q4 & Reference & - & Reference & - \\
\hline Estimated lifelong exposure & & $\mathrm{p}=0.002$ & & $p=0.049$ \\
\hline Low & $1.019(0.660-1.572)$ & 0.911 & $1.239(0.795-1.931)$ & 0.203 \\
\hline Below average & $1.396(0.972-2.003)$ & 0.017 & $1.340(0.884-2.032)$ & 0.066 \\
\hline Average & $1.342(0.940-1.916)$ & 0.032 & $1.350(0.924-1.974)$ & 0.039 \\
\hline Above average & $0.931(0.663-1.307)$ & 0.575 & $0.954(0.664-1.372) p$ & 0.734 \\
\hline High & Reference & - & Reference & - \\
\hline Self-perceived consistency of milk consumption & & $\mathrm{p}=0.004$ & & $p=0.018$ \\
\hline Never been drinking milk $\geq 5$ times/week & $1.289(0.957-1.736)$ & 0.027 & $1.376(0.980-1.933)$ & 0.015 \\
\hline Sometimes but not always & $1.359(1.065-1.733)$ & 0.001 & $1.252(0.965-1.624)$ & 0.025 \\
\hline Have been drinking milk $\geq 5$ times/week for most or all of my life & Reference & - & Reference & - \\
\hline
\end{tabular}

OR, odds ratio; CI, confidence interval; $Q$, quartile. *Also adjusted for energy intake for variables containing current milk and total dairy intake. Bold indicates statistical significance $(p<0.01)$.

previous clinical observations suggesting that high lactose intake was related to cataract risk in subjects with low activity of galactose-metabolic enzymes, but not when high levels of enzymatic activity were present [24].

We also found no clear association between the quartiles of current daily milk or total dairy intake and cataract history, a finding which is similar to that reported in a casecontrol study examining a Greek sample [7]. Possibly, because the nutritional profile of milk is rich with various nutrients, some components may "neutralize" the cataractogenic effects of lactose/galactose. For example, in one experiment, coadministering whey along with purified lactose in rats inhibited the formation of cataract that was otherwise observed when fed purified lactose alone [25]. Moreover, consistent intake of milk may replace other less healthy dietary behaviors/patterns that could otherwise increase the risk of cataract [26].

Interestingly, we did find a significant reduction in cataract history risk when assessing milk intake on a continuous scale. While it is less often encountered in the literature, a protective effect of dairy is reported occasionally with a specific dairy product $[8,20]$ or, less commonly, for a specific cataract type [27]. In the recent study examining Mediterranean adults, skimmed yogurt intake (but not milk or other types of dairy products) was associated with reduced cataract risk [8]. The authors postulated that this protection could be mediated by reduction in cardiovascular risk factors, such as diabetes, or inflammation. Nonetheless, other dairy products, including milk, examined in the same populations failed to show any association with cataract risk $[8,20]$. Hence, the possibility of the observed effect being coincidental cannot be ruled out.

As alluded to earlier, there are interesting discrepancies between findings from older vs. more recent reports regarding the association between milk intake and cataract. Multiple factors could have played a role. References to data stemming from animal experimentation were common $[14,23,25]$, but these models required notably high intake of lactose (25-70\% of total dietary intake) that is unlikely to be applicable to the human diet [5]. Besides, different indicators of milk exposure were historically used to assess this relationship in humans. For example, one approach was to examine expression of galactosemic enzymes in RBCs and correlate it with cataract $[28,29]$. Another approach was geographic segregation of milk consumption, lactose malabsorption, and cataract prevalence [5]. In the latter approach, the concordance found in the segregation of regions of high milk consumption and lactose absorption with high cataract incidence may be appealing, but one must exercise caution to avoid assigning group-level indicators to individual-level outcomes (i.e., ecological bias) [30].

While each approach is bound to have some limitations, we herein examined a set of individual-level indicators of milk consumption to assess the outcome. Therefore, 
conclusions are not prone to ecological bias. In addition, previous research indicates that the individual-level approach, used in this study, is more resilient to model misspecification than ecological studies [31]. Other noteworthy strengths of this study are the inclusive, nationally representative sampling of the population and the use of dietary data collected through a standardized, prevalidated protocol [10]. Moreover, indicators used herein covered patterns of both current and past milk consumption in amount and frequency, respectively. The intent was to assess the relationship from multiple perspectives so that better conclusions could be reached.

Nonetheless, this study has some limitations. Because the milk-related indicators were self-reported, there is a potential for recall bias. In addition, indicators and outcomes were collected cross-sectionally, and thus, causality of any observed findings could not have been established. Another limitation is that the subtypes of cataracts in the studied participants were unknown. Previous reports suggested the presence of links between cortical cataract formation and osmotic stress, particularly at the initial stages [32]. On the other hand, this link seems to be less relevant to the agerelated nuclear cataracts, which are largely oxidative stressdriven [32]. Therefore, a slight association of milk consumption with certain subtypes of cataract cannot be ruled out [6]. Also, the indicators of early-life consumption covered the regularity of milk consumption without the exact amounts. Finally, the focus of indicators used in this study was on the intake amounts and consistency. In conceptual terms, factors affecting serum lactose/galactose (and galactitol) levels would be the amount and consistency of milk consumption, intestinal lactase activity, and galactose absorption [33]. In turn, factors modulating intralenticular galactose levels (and cataract risk) include serum galactose levels, diffusion rate across aqueous humor and lens capsule, activity of galactose pathway enzymes [2, 24], and activity of enzymes of sugar alcohol metabolism within lens tissue $[1,2]$. Therefore, from a holistic perspective, examining the sequence of factors in this pathway from lactose intake to intralenticular galactose levels would be necessary to better understand its relationship with cataract risk in the general population. While such investigation would be cost-prohibitive at the population level, further focused investigations that use this strategy are encouraged.

\section{Conclusion}

While the link may be more relevant to susceptible individuals, milk consumption does not appear to be associated with the development of age-related cataract in the general population.

\section{Data Availability}

The data generated and/or analyzed in the current study are available in the US National Center for Health Statistics repository.

\section{Conflicts of Interest}

The authors declare that they have no conflicts of interest.

\section{Acknowledgments}

The authors would like to thank Jiangxia Wang for her suggestion and acknowledge the Wilmer Biostatistics Core Grant (EY01765).

\section{Supplementary Materials}

Table S1: categorization of the composite lifelong milk exposure variable. (Supplementary Materials)

\section{References}

[1] Y. Ai, Z. Zheng, A. O’Brien-Jenkins et al., "A mouse model of galactose-induced cataracts," Human Molecular Genetics, vol. 9, no. 12, pp. 1821-1827, 2000.

[2] A. I. Coelho, M. E. Rubio-Gozalbo, J. B. Vicente, and I. Rivera, "Sweet and sour: an update on classic galactosemia," Journal of Inherited Metabolic Disease, vol. 40, no. 3, pp. 325-342, 2017.

[3] Y. Xu, Y. Li, L. Ma et al., "d-galactose induces premature senescence of lens epithelial cells by disturbing autophagy flux and mitochondrial functions," Toxicology Letters, vol. 289, pp. 99-106, 2018.

[4] A. Y. Lee, S. K. Chung, and S. S. Chung, "Demonstration that polyol accumulation is responsible for diabetic cataract by the use of transgenic mice expressing the aldose reductase gene in the lens," Proceedings of the National Academy of Sciences, vol. 92, no. 7, pp. 2780-2784, 1995.

[5] F. J. Simoons, "A geographic approach to senile cataracts," Digestive Diseases and Sciences, vol. 27, no. 3, pp. 257-264, 1982.

[6] R. Bhatnagar, Y. R. Sharma, R. B. Vajpayee et al., "Does milk have a cataractogenic effect?," Digestive Diseases and Sciences, vol. 34, no. 11, pp. 1745-1750, 1989.

[7] S. Theodoropoulou, E. Samoli, P. G. Theodossiadis et al., "Diet and cataract: a case-control study," International Ophthalmology, vol. 34, no. 1, pp. 59-68, 2014.

[8] L. Camacho-Barcia, M. Bullo, J. F. Garcia-Gavilan et al., "Dairy products intake and the risk of incident cataracts surgery in an elderly mediterranean population: results from the PREDIMED study," European Journal of Nutrition, vol. 58, no. 2, pp. 619-627, 2018.

[9] K. Michaëlsson, A. Wolk, H. Melhus, and L. Byberg, "Milk, fruit and vegetable, and total antioxidant intakes in relation to mortality rates: cohort studies in women and men," American Journal of Epidemiology, vol. 185, no. 5, pp. 345-361, 2017.

[10] J. M. Conway, L. A. Ingwersen, and A. J. Moshfegh, “Accuracy of dietary recall using the USDA five-step multiple-pass method in men: an observational validation study," Journal of the American Dietetic Association, vol. 104, no. 4, pp. 595-603, 2004.

[11] My Pyramid and Food Patterns Equivalents Database, Food Surveys Research Group, United States Department of Agriculture, https://www.ars.usda.gov/northeast-area/beltsville-mdbhnrc/beltsville-human-nutrition-research-center/food-surveysresearch-group/docs/fped-methodology/.

[12] US Census Bureau, "Poverty thresholds," 2019, https://www. census.gov/data/tables/time-series/demo/income-poverty/historical-poverty-thresholds.html.

[13] National Center for Health Statistics, Survey Methods and Analytic Guidelines, National Health and Nutrition Examination Survey,https://wwwn.cdc.gov/nchs/nhanes/analyticguidelines. aspx-estimation-and-weighting-procedures. 
[14] H. S. Mitchell and W. M. Dodge, "Cataract in rats fed on high lactose rations," The Journal of Nutrition, vol. 9, no. 1, pp. 37-49, 1935.

[15] F. D. McAuley, "Cataracts in galactosaemia," British Journal of Ophthalmology, vol. 37, no. 11, pp. 655-660, 1953.

[16] C. P. Richter and J. R. Duke, "Cataracts produced in rats by yogurt," Science, vol. 168, no. 3937, pp. 1372-1374, 1970.

[17] R. E. Stevens, M. B. Datiles, S. K. Srivastava, N. H. Ansari, A. E. Maumenee, and W. J. Stark, "Idiopathic presenile cataract formation and galactosaemia," British Journal of Ophthalmology, vol. 73, no. 1, pp. 48-51, 1989.

[18] D. Stambolian, V. Scarpino-Myers, R. C. Eagle Jr., B. Hodes, and H. Harris, "Cataracts in patients heterozygous for galactokinase deficiency," Investigative Ophthalmology \& Visual Science, vol. 27, no. 3, pp. 429-433, 1986.

[19] C. Vettorazzi, D. Canales, F. Rosales et al., "Milk, lactose and ethanol as dietary factors in cataractogenesis in Guatemala. A case-control study," International Journal of Food Sciences and Nutrition, vol. 43, no. 3, pp. 155-162, 1992.

[20] A. Tavani, E. Negri, and C. La Vecchia, "Food and nutrient intake and risk of cataract," Annals of Epidemiology, vol. 6, no. 1, pp. 41-46, 1996.

[21] X. Cui, P. Zuo, Q. Zhang et al., "Chronic systemic D-galactose exposure induces memory loss, neurodegeneration, and oxidative damage in mice: protective effects of R-a-lipoic acid," Journal of Neuroscience Research, vol. 84, no. 3, pp. 647-654, 2006.

[22] J. H. Kinoshita, "Mechanisms initiating cataract formation. Proctor lecture," Investigative Ophthalmology, vol. 13, no. 10, pp. 713-724, 1974.

[23] Y. Chen, Y. X. Jiang, L. Yi et al., "Excessive milk intake as a risk factor, probably associated with oxidative stress, in experimental naphthalene-initiated cataract in rats," Ophthalmic Research, vol. 47, no. 2, pp. 87-97, 2012.

[24] P. F. Jacques, J. Phillips, S. C. Hartz, and L. T. Chylack, "Lactose intake, galactose metabolism and senile cataract," Nutrition Research, vol. 10, no. 3, pp. 255-265, 1990.

[25] S. Tamura and S. Tsuzuki, "Studies on the promoting factors of galactose and lactose metabolism in the whey iii. concerning substances in whey that inhibit cataract developed in rats on hypergalactose and hyperlactose diets," The Japanese Journal of Pharmacology, vol. 21, no. 1, pp. 97-106, 1971.

[26] P. N. Appleby, N. E. Allen, and T. J. Key, "Diet, vegetarianism, and cataract risk," The American Journal of Clinical Nutrition, vol. 93, no. 5, pp. 1128-1135, 2011.

[27] S. Miglior, P. E. Marighi, M. Musicco, C. Balestreri, A. Nicolosi, and N. Orzalesi, "Risk factors for cortical, nuclear, posterior subcapsular and mixed cataract: a case-control study," Ophthalmic Epidemiology, vol. 1, no. 2, pp. 93-105, 1994.

[28] E. Beutler, F. Matsumoto, W. Kuhl et al., "Galactokinase deficiency as a cause of cataracts," New England Journal of Medicine, vol. 288, no. 23, pp. 1203-1206, 1973.

[29] H. W. Skalka and J. T. Prchal, "Presenile cataract formation and decreased activity of galactosemic enzymes," Archives of Ophthalmology, vol. 98, no. 2, pp. 269-273, 1980.

[30] S. Greenland and H. Morgenstern, "Ecological bias, confounding, and effect modification," International Journal of Epidemiology, vol. 18, no. 1, pp. 269-274, 1989.

[31] M. Nurminen, "Linkage failures in ecological studies," World Health Statistics Quarterly. Rapport Trimestriel De Statistiques Sanitaires Mondiales, vol. 48, no. 2, pp. 78-84, 1995.
[32] P. J. Donaldson, A. C. Grey, B. Maceo Heilman, J. C. Lim, and E. Vaghefi, "The physiological optics of the lens," Progress in Retinal and Eye Research, vol. 56, pp. e1-e24, 2017.

[33] I. Birlouez-Aragon, L. Ravelontseheno, B. Villate-Cathelineau, G. Cathelineau, and G. Abitbol, "Disturbed galactose metabolism in elderly and diabetic humans is associated with cataract formation," Journal of Nutrition, vol. 123, no. 8, pp. 1370-1376, 1993. 